Trans

continentales
Transcontinentales

Sociétés, idéologies, système mondial

$8 / 9 \mid 2010$

Des migrations aux circulations transnationales

\title{
Les Irakiens en Syrie et en Jordanie
}

Régimes d'entrée et de séjour et effets sur les configurations migratoires Iraqis in Syria and Jordan. The effect of entry and residence regimes on migratory configurations

\section{Géraldine Chatelard et Mohamed Kamel Dorai}

\section{OpenEdition \\ Journals}

Édition électronique

URL : http://journals.openedition.org/transcontinentales/794

DOI : $10.4000 /$ transcontinentales.794

ISBN : 978-2-7351-1557-0

ISSN : 1775-397X

Éditeur

Editions de la maison des sciences de l'homme

Édition imprimée

Date de publication : 31 décembre 2010

ISSN : 1950-1684

Référence électronique

Géraldine Chatelard et Mohamed Kamel Dorai, « Les Irakiens en Syrie et en Jordanie »,

Transcontinentales [En ligne], 8/9 | 2010, document 9, mis en ligne le 31 décembre 2010, consulté le 08 septembre 2020. URL : http://journals.openedition.org/transcontinentales/794 ; DOI : https://doi.org/ 10.4000/transcontinentales.794

Ce document a été généré automatiquement le 8 septembre 2020

Tous droits réservés 


\section{Les Irakiens en Syrie et en Jordanie}

Régimes d'entrée et de séjour et effets sur les configurations migratoires

Iraqis in Syria and Jordan. The effect of entry and residence regimes on

migratory configurations

Géraldine Chatelard et Mohamed Kamel Dorai

1 Depuis la chute du régime de Saddam Hussein, en 2003, et les changements politiques qui ont suivi, les flux migratoires irakiens ont été fortement modifiés. La Syrie et la Jordanie voisines connaissent l'afflux de réfugiés et les circulations migratoires les plus importants. Pour les réfugiés, ces deux pays sont à la fois des lieux d'asile territorial, des espaces de transit mais aussi des zones d'installation sur le moyen terme. Les villes, et en particulier les capitales Damas et Amman, sont les principaux centres où se regroupent les différentes catégories de migrants, y compris ceux enregistrés auprès du Haut commissariat aux réfugiés (HCR), l'agence des Nations unies pour les réfugiés, en l'absence de lieux de regroupement imposés par les autorités.

2 La nature de l'émigration irakienne est beaucoup plus complexe qu'un flux de réfugiés et s'apparente à ce que les agences internationales qualifient de migration mixte. D'une part, l'arrivée des réfugiés ne s'est pas produite en une ou deux vagues principales, mais elle s'est construite sur la durée, avec des temps d'exil ponctués parfois de retours puis de départs définitifs. D'autre part, les circulations migratoires des Irakiens entre l'Irak et la Syrie ou la Jordanie sont intenses, tout comme les circulations de biens, de marchandises, de capitaux, d'informations et les échanges symboliques. Damas et Amman jouent en effet aussi le rôle de bases arrières ou de «frontières " sécurisées de l'Irak pour les entrepreneurs, pour les élites politiques et professionnelles, et pour d'autres catégories privilégiées de la population irakienne qui ont accès à la mobilité, au point que l'on peut parler, pour cette catégorie de migrants, de circulation transfrontalière régulières entre Bagdad et Amman ou Damas. La réalité est encore plus difficile à saisir si l'on considère que beaucoup, parmi les migrants irakiens, sont à la fois des réfugiés au sens sociologique du terme et des personnes engagées dans, ou aspirant à, des circulations transfrontalières. Enfin, aussi bien en Syrie qu'en Jordanie, on peut noter l'importance des liens que les réfugiés et autres migrants entretiennent 
avec les diasporas irakiennes plus lointaines. Damas et Amman sont des points de rencontre de la diaspora avec ceux qui ont quitté l'Irak récemment ou qui y résident encore, tandis que les liens avec les diasporas sont aussi entretenus par les circulations de capitaux et d'informations, cependant plus libres depuis la capitale jordanienne.

3 Plusieurs facteurs expliquent les tendances migratoires actuelles. En premier lieu, le contexte d'insécurité sur le territoire irakien, qui varie depuis 2003 et qui pèse inégalement sur les individus selon les régions ou les catégories sociales. Ensuite, la combinaison de deux préoccupations chez les migrants irakiens: la sécurité pour la personne et les membres de la famille face à la violence et aux difficultés économiques en Irak, mais également le souci de préserver l'unité de la cellule familiale en migration et d'accéder à des ressources économiques. La mobilité entre plusieurs pays est souvent une condition essentielle pour combiner au mieux ces préoccupations.

Enfin, les modalités d'accès et de séjour dans les pays voisins et d'émigration secondaire sont primordiales pour l'architecture des flux migratoires. En effet, la frontière entre la Jordanie et l'Irak est militarisée par les Jordaniens depuis les années 1990. D'autre part, la frontière entre la Syrie et l'Irak a été fortifiée par les Syriens depuis 2006, c'est-à-dire l'année même où l'afflux d'Irakiens a été le plus important (ICG 2008). Ces mesures de sécurisation des frontières ont pour effet de rendre les passages irréguliers extrêmement difficiles, et donc de limiter drastiquement les circulations non officielles de migrants ${ }^{1}$. C'est pourquoi cet article se concentre sur les régimes d'entrée et de séjour et n'aborde pas les migrations irrégulières, qui n'affectent les configurations migratoires irakiennes dans la région que très marginalement.

5 La Syrie et la Jordanie ont plusieurs points communs quant à l'accueil des Irakiens sur leur sol. Toutes deux sont de longue date des pays de transit et d'installation plus ou moins longue pour les Irakiens. Par ailleurs, ces deux pays, même s'ils accueillent - ou ont accueilli - différentes populations réfugiées sur leur sol (en particulier un grand nombre de Palestiniens), ne sont pas signataires de la convention de Genève de 1951 sur les réfugiés et n'ont pas de politique d'asile particulière ni de procédure de détermination du statut de réfugié. Tant en Syrie qu'en Jordanie, les modalités d'entrée et de séjour des Irakiens sont fondées sur le régime général de l'immigration, avec la mise en place progressive d'un système spécifique pour les ressortissants irakiens visant à contrôler les entrées et limiter la durée du séjour, comme on le verra cidessous. Au-delà de ces similarités, la gestion du flux migratoire irakien par ces deux États est très contrastée pour des raisons de géopolitique et d'économie politique.

\section{La Syrie, de la libre circulation à l'ouverture sélective de la frontière}

6 Bien que la frontière syro-irakienne ait été officiellement fermée entre 1982 et 1997, quelques dizaines de milliers d'Irakiens se sont exilés en Syrie à la suite de la guerre du Golfe (1990-1991) et tout au long des années 1990 en raison du contexte politique mais aussi des difficultés économiques générées par l'embargo international sur l'Irak. Même si quelques milliers de personnes ont poursuivi leur émigration, le HCR estime qu'en 2003, avant le conflit en Irak, 60000 à 70000 Irakiens résidaient en Syrie. Un mouvement de retour a été amorcé entre la chute du régime de Saddam Hussein et 2004, date à laquelle de nouveaux réfugiés sont venus trouver asile en Syrie: tout 
d'abord des membres du parti Ba'th anciennement au pouvoir, puis des citadins, surtout en provenance de Bagdad, fuyant les attaques ciblées contre les intellectuels et les membres des professions libérales ou la criminalité généralisée puis, à partir du début de l'année 2006, l'augmentation des violences interconfessionnelles. Les premiers arrivés étaient des personnes plutôt aisées qui pensaient s'y installer pour une période limitée. Ils se sont assurés un permis de séjour en Syrie en investissant dans le commerce, l'industrie ou l'immobilier, ou en trouvant un emploi dans le secteur tertiaire, en particulier l'éducation supérieure. À partir de 2006, de plus en plus d'Irakiens défavorisés sont venus chercher refuge en Syrie. Issus aussi bien des zones urbaines que des zones rurales, ils sont arrivés sans capital économique, et avec beaucoup moins de bagage éducatif ou professionnel que le groupe précédent.

7 Entre le début du conflit irakien, au printemps 2003, et le début de l'année 2007, la Syrie a ouvert sa frontière aux Irakiens en leur offrant le même régime d'entrée et de séjour très souple qu'aux autres ressortissants arabes: un visa d'entrée universel obtenu à la frontière, la possibilité de séjourner par périodes de trois mois renouvelables en sortant et en entrant à nouveau immédiatement dans le pays, le tout en accédant aux services sociaux (éducation, santé) et à la possibilité de chercher du travail sur place. Un contrat de travail ou des investissements, comme l'achat d'un logement, ouvrent le droit à un séjour d'un an renouvelable. Cependant, seule la minorité d'Irakiens disposant de capital important a ainsi pu sécuriser son séjour, tandis que le marché de l'emploi était saturé et protectionniste.

8 En 2007, les conséquences de la mise en place d'un visa sélectif et payant ${ }^{2}$ ont été multiples. D'une part, le nombre d'entrées des Irakiens a largement diminué. D'autre part, les circulations entre la Syrie et l'Irak ont aussi connues une baisse. De plus, un certain nombre d'Irakiens parmi les plus pauvres et résidant en Syrie sans titre de séjour sont repartis en Irak plutôt que de demeurer en situation irrégulière. La seule infraction au séjour ne constitue pas un motif de reconduite à la frontière. Une des conséquences principales de l'instauration de ce visa a été de créer un groupe de personnes, résidant de fait en Syrie, mais dans l'impossibilité de légaliser leur situation ou de se déplacer entre la Syrie et l'Irak. En effet, parmi ceux qui sont arrivés en Syrie avant l'introduction des nouvelles mesures et qui ne bénéficient pas de titres de séjour, beaucoup ne peuvent ou ne veulent pas retourner en Irak de façon durable. Ils n'ont pas les moyens de couvrir les frais induits par le renouvellement mensuel d'un visa et l'achat des justificatifs nécessaires, qu'il est possible d'obtenir sur le marché noir en Syrie ou en Irak à défaut d'appartenir réellement aux catégories autorisées.

9 L'État syrien n'a ni les capacités ni la volonté d'expulser les dizaines de milliers d'Irakiens en situation irrégulière sur son territoire. Ces mesures d'entrée sélectives sont cependant bien destinées à endiguer de nouveaux flux d'Irakiens aux revenus les plus modestes et à inciter les personnes en situation irrégulière à quitter le pays, le tout dans un contexte de crise économique et d'inflation aiguë où une large partie des Irakiens est en compétition avec les Syriens pour les ressources et les emplois informels. 


\section{La Jordanie, une circulation migratoire sous contrôle croissant}

10 Entre la guerre du Golfe de 1990-1991 et la chute du régime ba'thiste au printemps 2003, la Jordanie a été le seul pays voisin de l'Irak à autoriser sans condition l'accès des Irakiens sur son territoire par un visa d'entrée de trois mois obtenu à la frontière et renouvelable. Personnes, biens et capitaux circulaient dans les deux sens. Indépendamment du changement de régime survenu en Irak en 2003, trois types de flux migratoire sont à distinguer : les circulations de l'élite économique et politique qui vient pour accéder à des offres de services indisponibles dans son pays (soins médicaux de qualité, système bancaire international, etc.) ou à des marchés inaccessibles du fait de l'embargo international avant 2003 et de l'insécurité depuis; les migrations de travail de la classe moyenne éduquée vers la Jordanie ou vers d'autres pays arabes importateurs de main-d'œuvre qualifiée via la Jordanie; et l'arrivée des réfugiés qui fuient la répression exercée par le régime ou les violences locales. Sous le régime de Saddam Hussein, qui exerçait un contrôle sur la mobilité internationale de ses ressortissants, ce n'était pas l'entrée en Jordanie qui était problématique, mais la sortie d'Irak. Il fallait bénéficier d'un fort capital financier ou relationnel pour contourner les restrictions et même pouvoir payer des faux papiers. Entre 1991 et 2003, la Jordanie a sans doute été le principal pays de transit du demi-million d'Irakiens qui ont déposé une demande d'asile sur le territoire ou à l'ambassade d'un pays occidental, qui ont bénéficié des programmes de réinstallation du HcR, ou qui ont migré via le regroupement familial. Selon certaines estimations, il y avait fin 2002 environ 300000 Irakiens en Jordanie - soit résidents, soit en transit ou en visite.

11 Les restrictions sur la sortie ont été levées avec la chute de Saddam Hussein. Entre 2003 et 2004, certains des Irakiens de Jordanie, comme leurs compatriotes de Syrie, ont opéré un mouvement de retour. Au même moment, d'autres quittaient l'Irak pour des motifs politiques, familiaux ou personnels. Certains transitaient par la Jordanie vers d'autres pays arabes ou occidentaux. D'autres s'installaient plus durablement en obtenant un titre de séjour grâce à des investissements ou un contrat de travail. D'autres, enfin, ont renouvelé leur visa de touriste tous les trois mois dans l'attente des évolutions de la situation en Irak ou des perspectives d'émigration plus lointaine.

À la suite des attentats perpétrés par des Irakiens à Amman, en novembre 2005, les conditions d'entrée et de séjour ont été progressivement durcies pour les ressortissants d'Irak. Dans un premier temps, un filtrage aux frontières a rendu l'entrée des Irakiens beaucoup moins systématique ; ont été surtout refoulés les individus identifiés comme chiites et les hommes entre 16 et 35 ans. La durée du séjour a été réduite à un mois, parfois moins. En janvier 2008, la Jordanie a mis en place un visa sélectif selon des critères similaires à ceux mis en place par la Syrie. Depuis début 2006, le nombre d'entrées en Jordanie a considérablement baissé alors même qu'au début de cette année-là, les violences interconfessionnelles avaient augmenté le nombre de déplacements forcés à l'intérieur et hors d'Irak. L'immense majorité des Irakiens actuellement en Jordanie appartient à la classe moyenne éduquée et citadine, en particulier originaire de Bagdad, tandis que la proportion de chiites est bien moins élevée qu'en Syrie. Les circulations des élites politiques, économiques et professionnelles irakiennes entre Bagdad et Amman continuent sans entraves, tout comme celles des Irakiens qui viennent étudier ou se faire soigner en Jordanie. 
Contrairement à ce qui s'est passé en Syrie, la création du visa sélectif n'a pas engendré de retours importants, l'entrée étant déjà largement contrôlée avant la mise en place de cette mesure. Cependant, comme en Syrie, cette mesure a créé de l'irrégularité et du confinement. L'absence de statut légal et de perspectives économiques, et l'impossibilité de déplacement vers l'Irak, ont poussé de nombreux Irakiens à quitter la Jordanie en activant leurs réseaux dans la diaspora, en épuisant leurs ressources financières pour payer des passeurs, ou en se présentant au HCR en vue d'une réinstallation. Cela alors même qu'ils envisageaient leur séjour comme une période d'attente en sécurité avant un éventuel retour en Irak.

Les restrictions sur les entrées mises en place par la Jordanie dès 2005 ont donc eu pour effet de rediriger le flux des réfugiés vers la Syrie, d'opérer le tri des migrants sur des bases confessionnelles, économiques et professionnelles, et de modifier la distribution démographique des flux migratoires au niveau régional. La Jordanie cède dès lors à la Syrie la place de premier pays arabe de la région en termes de flux et de séjour de migrants irakiens.

\section{Des régimes migratoires qui limitent la mobilité transfrontalière}

Dans les deux pays, les régimes d'entrée sélectifs pénalisent les Irakiens les plus vulnérables en termes de sécurité personnelle et économique et ont des effets notables sur les flux et les configurations migratoires. Ils empêchent, par exemple, les personnes qui subissent l'insécurité et la violence mais qui ne disposent pas de capital financier ou social de sortir d'Irak. Dans les pays d'accueil, ils pénalisent les migrants déjà entrés et poussent dans l'irrégularité les Irakiens les plus défavorisés qui ne peuvent maintenir des circulations transfrontalières pour régulariser leur séjour.

16 La politique de visa, en limitant les possibilités de circulation, pénalise également les Irakiens qui maintiennent des liens avec leur pays par une activité économique, soit pour assurer la survie de leurs familles réfugiées en Jordanie ou Syrie, soit dans la perspective d'un retour. Face aux difficultés de circulation, de plus en plus d'Irakiens vendent les biens, en particulier immobiliers, qu'ils avaient conservés en Irak. Cela a pour conséquence de les immobiliser dans leur pays d'accueil, non pas parce qu'ils ne peuvent pas retourner en Irak, mais parce qu'ils craignent de ne pas être admis à retourner dans un pays voisin. Ces régimes ont également pour effet de rendre plus durable la séparation des familles entre plusieurs pays de la région. De ce fait, et bien que ce ne soit pas le seul facteur qui motive ces projets, la difficulté croissante pour certains à circuler entre l'Irak et les pays voisins peut être un élément important motivant la décision de chercher un exil durable en Europe, en Amérique du Nord ou en Australie.

\section{Les migrations secondaires face aux restrictions des pays industrialisés}

17 Les parcours migratoires des Irakiens sont le plus souvent complexes et constitués de plusieurs étapes, ce qui contraint les réfugiés à adopter des stratégies d'adaptation pour faire face aux difficultés qu'ils rencontrent au fur et à mesure de leur itinéraire. La 
majeure partie des familles rencontrées ont été séparées sur des périodes plus ou moins longues. Pour certaines le Proche-Orient est une simple étape, un premier pays où les réfugiés trouvent la sécurité et tentent de poursuivre leur parcours vers un pays tiers pour s'y installer sur le long terme. Cette stratégie d'un exil par étapes est souvent un passage obligé pour les réfugiés quand les organisations internationales sont limitées dans leur activité à cause de la violence généralisée, et parce que les pays voisins, premier espace d'accueil, ne proposent pas de solutions durables en termes d'installation, de séjour ou d'emploi. Une fois arrivés dans leur premier pays d'accueil, les réfugiés sollicitent leurs réseaux familiaux sur place et en diaspora pour obtenir de l'aide financière et préparer leur émigration. Ils s'installent dans un provisoire précaire durant lequel ils organisent la poursuite de leur parcours.

18 Au regard du nombre d'Irakiens accueillis dans les pays voisins, la majeure partie des pays industrialisés - à l'exception notable de la Suède jusqu'en 2008 - ont fermé leurs portes aux réfugiés et demandeurs d'asile irakiens, en leur octroyant au comptegouttes le statut de réfugié. L'Union européenne est la principale destination de ces réfugiés (plus de la moitié des demandes d'asile dans les pays industrialisés), même si des pays comme l'Australie, les États-Unis ou le Canada en accueillent également quelques milliers sous divers statuts.

L'évolution récente de ces demandes d'asile montre que les principaux pays d'accueil (Suède et Grèce) ont instauré des politiques pour limiter l'arrivée des réfugiés irakiens. D'autres pays, tels que l'Allemagne et la France, ont mis en place des programmes spécifiques visant l'accueil d'un nombre limité de réfugiés sélectionnés au préalable.

Tableau 1

\begin{tabular}{|l|r|r|}
\hline Pays & $\mathbf{2 0 0 7}$ & $\mathbf{2 0 0 8}$ \\
\hline Allemagne & 4171 & 6697 \\
\hline Belgique & 825 & 1070 \\
\hline France & 144 & 627 \\
\hline Grèce & 5474 & 1760 \\
\hline Norvège & 1227 & 3137 \\
\hline Pays-Bas & 2004 & 5027 \\
\hline Suède & 18559 & 6083 \\
\hline Suisse & 935 & 1440 \\
\hline Royaume-Uni & 2075 & 1530 \\
\hline
\end{tabular}

Nombre de nouvelles demandes d'asile d'Irakiens dans une sélection de pays européens (2007-2008). Source: UNHCR, New Asylum Applications Lodged in Selected Countries in Europe, North America, Oceania and Asia, 30 janvier 2009. 
chiffres relativement limités sont le résultat des politiques restrictives mises en œuvre par de nombreux pays européens qui combinent un accès à la demande d'asile rendu difficile par l'assimilation des demandeurs potentiels à des migrants illégaux et des taux de reconnaissance très faibles destinés à décourager les nouvelles arrivées.

Dans ce contexte de maintien et/ou de radicalisation des politiques instaurées par les pays développés pour contenir l'arrivée des demandeurs d'asile irakiens sur leur sol, les politiques de réinstallation préconisées par le HCR demeurent elles aussi limitées et ne concernent qu'une infime fraction des réfugiés présents en Syrie et en Jordanie. Depuis février 2007, 19000 réfugiés ont été réinstallés dans des pays tiers, dont un peu plus de 13000 aux États-Unis (UNHCR Syria at a Glance, avril 2010, Damas), ces chiffres étant très nettement inférieurs au nombre de personnes auxquelles le HCR a proposé la réinstallation.

Tableau 2

\begin{tabular}{|l|l|}
\hline Année & Total \\
\hline 2004 & 8384 \\
\hline 2005 & 10829 \\
\hline 2006 & 19496 \\
\hline 2007 & 38107 \\
\hline 2008 & 26195 \\
\hline
\end{tabular}

Demandeurs d'asile irakiens dans I'Union européenne (2004-2008)

Face à l'instabilité qui perdure en Irak et à la fermeture des portes de nombreux pays occidentaux aux demandeurs d'asile, donc à la difficulté pour les Irakiens de voyager, les regroupements familiaux se font souvent à Damas ou à Amman, où se multiplient les mariages avec des Irakiens de la diaspora venus d'Australie, du Canada, des États-Unis, de Suède ou d'Allemagne. Leurs conjoints ou conjointes les rejoignent ensuite par le biais du regroupement familial. Mais, dans l'exil, les écarts se creusent tout autant entre les familles qui ont d'importantes connexions locales et/ou transnationales et celles qui ont un accès limité aux ressources et à la mobilité.

\section{Conclusion}

Les régimes gouvernant l'entrée et le séjour des Irakiens aussi bien en Syrie qu'en Jordanie ont pour effet de créer des différentiations sociales et spatiales. D'une part, ces régimes jouent un rôle dans la distribution actuelle des migrants irakiens entre les deux pays en termes de volume et de profil socioéconomique. D'autre part, en créant différentes catégories de migrants, ces mesures induisent un différentiel d'accès à la sécurité via la mobilité, produisent de nouvelles stratifications sociales, et forcent les migrants les moins privilégiés à adapter leurs projets migratoires. La diversité des expériences migratoires, leur inscription dans des temporalités plus ou moins longues 
et leurs logiques plurielles (asile, refuge temporaire, transit, commerce, pèlerinage) caractérisent aujourd'hui la migration irakienne en Syrie en Jordanie. La majeure partie des Irakiens peuvent être considérés comme réfugiés, mais l'absence de reconnaissance de ce statut par les autorités des pays d'accueil rend leur installation précaire et réversible. Si les plus dotés en capital économique et social ont réussi à stabiliser leur situation juridique et/ou socioprofessionnelle dans leur pays d'accueil, beaucoup connaissent une baisse importante de leur niveau de vie et ne peuvent trouver du travail. Ce facteur, couplé à la difficulté de maintenir des liens tangibles avec l'Irak et l'intégrité de la cellule familiale par la circulation transfrontalière, incite un grand nombre d'Irakiens à chercher un nouveau pays d'accueil pour s'installer durablement, et à tenter d'émigrer de façon légale ou illégale même si les principaux pays d'accueil hors du Moyen-Orient tendent à fermer leurs portes.

\section{BIBLIOGRAPHIE}

CHATELARD, G. 2010. « What visibility conceals. Re-embedding refugee migration from Iraq », in D. CHATTY et B. FINLAYSON (dir.), Dispossession and Displacement. Forced Migration in the Middle East and North Africa. New York, Oxford University Press : 17-44.

CHATELARD, G. 2010. "Cross-Border mobility of Iraqi refugees », Forced Migration Review, $34: 60-61$ [http://www.fmreview.org/urban-displacement/FMR34/60-61.pdf].

CHATELARD, G. 2010. « Jordan. A refugee haven », Migration Information Source, Washington DC, Migration Policy Institute [http://www.migrationinformation.org/Profiles/display.cfm?ID=794]. CHATELARD, G. 2009. « Migration from Iraq between the Gulf and the Iraq wars (1990-2003) : Historical and sociospacial dimensions ", Working Paper 09-68, COMPAS - Centre on Migration, Policy and Society (Université d'Oxford) [http://www.compas.ox.ac.uk/publications/workingpapers/wp-09-68/\#c221].

CHATELARD, G. et K. M. DORAÏ. 2009. « La présence irakienne en Syrie et en Jordanie. Dynamiques sociales et spatiales et mode de gestion par les pays d'accueil », Maghreb-Machrek, 199 (numéro spécial Les Migrations au Proche-Orient) : 43-60.

DORAÏ, M. K. 2010. «Les Irakiens en Suède. Exil et recomposition migratoire depuis la chute du régime de Saddam Hussein en 2003 ", e-migrinter, 5 : 13-23 [http://www.mshs.univ-poitiers.fr/ migrinter/e-migrinter/201005/e-migrinter2010_05_013.pdf].

DORAÏ, M. K. 2009. «Le Moyen-Orient, principal espace d'accueil des réfugiés irakiens », in MIGREUROP, Atlas des migrants en Europe. Géographie critique des politiques migratoires. Paris, Armand Colin : 109-111.

DORAÏ, M. K. 2009. « Le rôle de la Syrie dans l'accueil des réfugiés Irakiens depuis 2003 : espace de transit, espace d'installation ", Méditerranée, $113: 139-146$.

IcG. 2008. Failed Responsibility. Iraqi Refugees in Syria, Jordan and Lebanon, International Crisis Group, Middle East Report, 77, 41 p. [http://www.crisisgroup.org/ /media/Files/ 
Middle\%20East\%20North\%20Africa/Iraq\%20Syria\%20Lebanon/Iraq/

ailed_responsibility__iraqi_refugees_in_syria__jordan_and_lebanon.ashx].

SPERL, M. 2007. « Fortress Europe and the Iraqi “intruders" : Iraqi asylum-seekers and the EU, 2003-2007 ", in New Issues in Refugee Research. Genève, UNHCR Policy Development and Evaluation Service (Research Paper ; 144), 19 p. [http://www.unhcr.org/publ/RESEARCH/470c9be92.pdf].

\section{NOTES DE FIN}

1. Cette situation contraste avec celle d'autres frontières dans la région du Moyen-Orient qui sont peu ou moins contrôlées, par exemple la frontière entre le Kurdistan irakien et la Turquie, ou celle entre la Syrie et le Liban.

2. Au premier semestre 2007 , face à l'afflux d'Irakiens qui fuyaient les violences diverses et l'insécurité économique, la Syrie a amorcé un changement de ses politiques d'entrée et de séjour, sans toutefois fermer totalement l'accès à son territoire. La durée du visa d'entrée accordé aux Irakiens à la frontière a d'abord été réduite à un mois renouvelable par sortie et ré-entrée. Puis, fin 2007, l'entrée a été restreinte à quinze catégories d'Irakiens sur présentation de pièces justificatives : visites pour des raisons professionnelles, pour études ou pour traitement médical ; les conducteurs de camion et de taxi qui font la navette entre la Syrie et l'Irak; les Irakiens possédant une résidence dans un pays tiers, ou ceux en transit vers un pays tiers ou pour rentrer en Irak. Ce visa d'un mois peut actuellement s'obtenir à la frontière sur présentation de justificatifs et avec un paiement de 50 dollars américains.

\section{RÉSUMÉS}

Le conflit actuel en Irak a été et continue d'être le moteur de déplacements de grande ampleur d'individus, de familles et de groupes sociaux entiers, tels les chrétiens. Dans le cas de l'Irak, ces déplacements peuvent prendre diverses formes, depuis l'évitement de certaines zones jusqu'à des mouvements de masse vers des lieux très éloignés, y compris au-delà des frontières. De manière générale, on sait peu de choses sur les processus de déplacements forcés. Ce projet est centré sur les circonstances et les temporalités de ces mouvements migratoires, avec une attention particulière portée aux processus de prise de décision, aux sources d'information disponibles, et au rôle des réseaux sociaux (familiaux, professionnels, communautaires, tribaux, etc.). Les réfugiés sont au centre de cette problématique en étant considérés comme des sujets actifs dans la migration dont les décisions et les choix sont contraints par les circonstances mais qui ont des aspirations et peuvent élaborer des stratégies. Dans quelle mesure les individus/ familles/groupes sociaux peuvent-ils exercer un choix quant à la temporalité et à la direction de leur migration? De quelles sources d'information disposent-ils (familles, communautés, membres de la diaspora, réseaux professionnels, médias, institutions publiques, religieuses, organisations internationales) ? Quelles sont leurs attentes en matière de sécurité en Irak et dans les autres pays? Comment s'opère le choix de la destination? Quels sont les éléments qui distinguent les déplacés internes des réfugiés au sens juridique (ayant traversé une frontière internationale)? Quels sont les facteurs qui influent sur la prise de décisions? Comment les relations de genre et intergénérationnelles au sein la famille affectent-elles la décision et la nature des mouvements des familles et des groupes sociaux? Les facteurs cumulatifs sont-ils un moteur de la migration de masse, en particulier pour des communautés religieuses entières comme les chrétiens? Y a-t-il un seuil au-delà duquel rester ou retourner n'est plus envisageable? Quelle est la nature des réseaux sociaux transnationaux et comment influencent- 
ils la décision du départ et de la poursuite la migration au-delà du premier lieu de déplacement (à l'intérieur ou hors de l'Irak)?

The current conflict in Iraq has caused and is still causing large-scale movements of individuals, families and entire social groups (such as Christians). In the Iraq context, these movements can take several forms, from avoiding certain areas to mass movements towards distant regions (including across borders). Generally speaking, we know little about forced displacement processes. This article deals with the circumstances leading to and timeframes of migratory movements, focusing on decision-making processes, available sources of information, and the role of social networks (families, professionals, communities, tribes, etc.). Refugees are at the heart of this issue because they are considered active subjects of migration. Their decisions and choices depend on circumstances, but they also have aspirations and can elaborate strategies. To what extent are individuals/families/social groups able to choose the timeframe and direction of their migration? What sources of information do they have (families, communities, diaspora members, professional networks, the media, public institutions, religious institutions or international organisations)? What are their expectations for security in Iraq and other countries? How do they choose their destinations? What are the legal differences separating internally displaced people from refugees (having crossed international borders)? Which factors influence decision-making? How do gender and intergenerational relations within families affect the decisions and movements of families and social groups? Do cumulative factors trigger mass migration, especially for religious communities such as Christians? Is there a point beyond which staying or going is no longer possible? What characterises transnational social networks? How do they inflence decisions to leave a place and decisions to migrate further than the first port of call (inside or outside Iraq)?

\section{INDEX}

Thèmes : migration, mobilité

Keywords : transnational social networks, migration strategy, refuge, Syria, Iraq, Jordan Index géographique : Syrie, Irak, Jordanie

Mots-clés : réseaux sociaux transnationaux, stratégie migratoire, réfugié

\section{AUTEURS}

\section{GÉRALDINE CHATELARD}

Chercheure associée dans le département des études contemporaines à l'Institut français du Proche-Orient (ifpo-umifre 6) à Amman, http://www.ifporient.org/ depuis 2006, g.chatelard@ifporient.org

\section{MOHAMED KAMEL DORAI}

Cnrs-Migrinter, Mohamed-Kamel.Dorai@univ-poitiers.fr 